\title{
Limits to the Accuracy of 3D Thickness Measurement in Magnetic Resonance Images
}

\author{
Yoshinobu Sato ${ }^{1}$, Katsuyuki Nakanishi ${ }^{2}$, Hisashi Tanaka ${ }^{2}$, Takashi Nishii ${ }^{3}$, \\ Nobuhiko Sugano ${ }^{3}$, Hironobu Nakamura ${ }^{2}$, Takahiro Ochi ${ }^{4}$, Shinichi Tamura ${ }^{1}$ \\ 1 Division of Interdisciplinary Image Analysis \\ 2 Department of Radiology \\ 3 Department of Orthopaedic Surgery \\ 4 Division of Computer Integrated Orthopaedic Surgery \\ Osaka University Graduate School of Medicine \\ yoshi@image.med.osaka-u.ac.jp, http://www.image.med.osaka-u.ac.jp/yoshi
}

\begin{abstract}
Measuring the thickness of sheet-like (or plate-like) anatomical structures, such as articular cartilages and brain cortex, in 3D magnetic resonance (MR) images is often an important diagnostic procedure. The purpose of this paper is to investigate the fundamental limits to the accuracy of thickness determination in MR images. Given imaging and postprocessing parameters, the characteristics of thickness determination accuracy are derived by means of a theoretical simulation method, focusing especially on the effect of sheet structure orientation on accuracy in the case of noncubic (anisotropic) voxels. The theoretical simulation was validated by in vitro experiments.
\end{abstract}

\section{Introduction}

Thickness measurement of sheet-like (or plate-like) anatomical structures in magnetic resonance (MR) images is an important diagnostic procedure in, for example, the diagnosis of joint diseases by evaluating the distribution of articular cartilage thicknesses [1], [2], [3], 4], 5], 6], [7], 8], or of specific neuropsychiatric disorders by assessing cortical thicknesses in the brain 9 , 10. While several methods for thickness quantification have been proposed [5], [6], [9], [8], accuracy limits arising from finite resolution have not been evaluated. Although Sato et al. examined accuracy limits due to partial volume averaging, especially the effect of anisotropic voxels, by software simulation 8], the modeling of MR image acquisition was insufficient and validation with actual MR images was not demonstrated.

The goal of the work described here is to provide a theoretical procedure for ascertaining the inherent limits to the accuracy of thickness determination in MR images. We especially address the manner in which how the accuracy depends on the orientation of sheet structures when a voxel shape is anisotropic. Sheet structures of interest are often distributed within specific orientation ranges - for example, on an approximately cylindrical surface. In this case, the resolution in the plane orthogonal to the axis of the approximated cylinder should be higher 
than that along the axis to maximize the accuracy of thickness measurement. Given the orientation distribution of sheet structures and the fixed volume of a voxel - which is directly related to the signal-to-noise ratio - the voxel shape can be optimized. In this paper, we define thickness as the distance between the outer and inner boundaries measured at subvoxel resolution, which are the zero-crossings of directional second derivatives along the normal directions of the sheet surface. We establish a method for evaluating the accuracy of thickness measurement by numerical simulation, and validate the method through in vitro experiments.

\section{Materials and Methods}

\subsection{Numerical Simulation}

Modeling a Sheet Structure A three-dimensional (3D) sheet structure orthogonal to the $x$-axis is modeled as

$$
S(\mathbf{x} ; t)=B(x ; t)
$$

where $\mathbf{x}=(x, y, z)$.

$$
B(x ; t)=\left\{\begin{array}{lr}
1, & -\frac{1}{2} t \leq x \leq \frac{1}{2} t \\
0, & \text { otherwise, }
\end{array}\right.
$$

in which $t$ represents the thickness of the sheet.

Let $\theta$ be the rotation angle of the sheet structure around the $y$-axis. The 3D sheet structure with rotation $\theta$ is written as

$$
S(\mathbf{x} ; t, \theta)=S_{0}\left(\mathbf{x}^{\prime} ; t\right),
$$

where $\mathbf{x}^{\prime}=R_{\theta} \mathbf{x}$, in which $R_{\theta}$ detnotes a $3 \times 3$ matrix representing rotation $\theta$ around the $y$-axis.

Modeling MR Image Acquisition The one-dimensional (1D) point spread function (PSF) of MR images [1] is given by

$$
M\left(x ; \Delta_{x}\right)=\frac{1}{N_{x}} \frac{\sin \left(\pi \frac{x}{\Delta_{x}}\right)}{\sin \left(\pi \frac{x}{N_{x} \Delta_{x}}\right)},
$$

where $N_{x}$ is the number of samples in the frequency domain, and $\Delta_{x}$ represents the sampling interval in the spatial domain. Eq. (4) is well-approximated [12] by

$$
M\left(x ; \Delta_{x}\right)=\frac{1}{N_{x}} \frac{\sin \left(\pi \frac{x}{\Delta_{x}}\right)}{\pi \frac{x}{\Delta_{x}}} .
$$

The 3D PSF is given by

$$
M\left(\mathbf{x} ; \Delta_{x}, \Delta_{y}, \Delta_{z}\right)=M\left(x ; \Delta_{x}\right) M\left(y ; \Delta_{y}\right) M\left(z ; \Delta_{z}\right)
$$

The MR image of the sheet structure with rotation $\theta$ and thickness $t$ is given by

$$
I(\mathbf{x})=S(\mathbf{x} ; t, \theta) * M\left(\mathbf{x} ; \Delta_{x}, \Delta_{y}, \Delta_{z}\right),
$$

where $*$ denotes the convolution operation. 
Theoretical Methods for Thickness Determination Thickness is determined by measuring the distance between boundaries corresponding to the inner and outer edges of a sheet structure, which are the zero-crossings of directional second derivatives along the normal vector of the sheet. The directional second derivatives are combined with Gaussian blurring. In actual situations, Gaussian blurring is typically employed to reduce the effect of noise. The partial second derivative combined with Gaussian blurring for the MR image $I(\mathbf{x})$, for example, is given by

$$
I_{x x}(\mathbf{x} ; \sigma)=\frac{\partial^{2}}{\partial x^{2}} G(\mathbf{x} ; \sigma) * I(\mathbf{x}),
$$

where $G(\mathbf{x} ; \sigma)$ is the isotropic 3D Gaussian function with the standard deviation $\sigma$.

Let $\nabla^{2} I(\mathbf{x} ; \sigma)$ be the Hessian matrix of $I(\mathbf{x} ; \sigma)$, which is given by

$$
\nabla^{2} I(\mathbf{x} ; \sigma)=\left[\begin{array}{lll}
I_{x x}(\mathbf{x} ; \sigma) & I_{x y}(\mathbf{x} ; \sigma) & I_{x z}(\mathbf{x} ; \sigma) \\
I_{y x}(\mathbf{x} ; \sigma) & I_{y y}(\mathbf{x} ; \sigma) & I_{y z}(\mathbf{x} ; \sigma) \\
I_{z x}(\mathbf{x} ; \sigma) & I_{z y}(\mathbf{x} ; \sigma) & I_{z z}(\mathbf{x} ; \sigma)
\end{array}\right]
$$

The directional second derivative along the normal direction of the sheet structure is given by

$$
D_{2}(\mathbf{x} ; \sigma, \mathbf{r})=\mathbf{r}^{\top} \nabla^{2} I(\mathbf{x} ; \sigma) \mathbf{r}
$$

in which $\mathbf{r}=(\cos \theta, 0, \sin \theta)$.

Similarly, the directional first derivative along the sheet normal is given by

$$
D_{1}(\mathbf{x} ; \sigma, \mathbf{r})=\mathbf{r}^{\top} \nabla I(\mathbf{x} ; \sigma)
$$

in which $\mathbf{r}=(\cos \theta, 0, \sin \theta)$ and $\nabla I(\mathbf{x} ; \sigma)$ is the gradient vector given by

$$
\nabla I(\mathbf{x} ; \sigma)=\left(I_{x}(\mathbf{x} ; \sigma), I_{y}(\mathbf{x} ; \sigma), I_{z}(\mathbf{x} ; \sigma)\right) .
$$

Both sides of the boundaries for sheet structures can be defined as the points having the maximum and minimum values of $D_{1}(\mathbf{x} ; \sigma, \mathbf{r})$ among those satisfying the condition given by $D_{2}(\mathbf{x} ; \sigma, \mathbf{r})=0$, where $\mathbf{r}=(\cos \theta, 0, \sin \theta)$. The distance between the two detected boundary points along the direction $\mathbf{r}$ is defined as the measured thickness, $T$.

Simulation Method Voxels in MR volume data are typically anisotropic since they usually have lower resolution along the third direction (orthogonal to the slice plane) than within slices. Hence, we assume that the resolution along the $z$-axis is lower than that in the $x y$-plane and that pixels in the $x y$-plane are square. Let $\Delta_{x y}\left(=\Delta_{x}=\Delta_{y}\right)$ be the pixel size within the slices and $\Delta_{x y} \leq \Delta_{z}$. We then determine the measured thickness, $T$, by the above-described numerical simulation under different combinations of $t, \theta, \Delta_{x y}, \Delta_{z}$, and $\sigma$. 


\subsection{In Vitro Experiments}

Materials and MR Image Acquisition To validate the numerical simulation method, we used a resected femoral head, approximately spherical in shape, with articular cartilages distributed on its surface. The cartilages were used as the material for the experiments, in which we assumed they were distributed on a spherical surface. The cartilage thickness was then measured along the normal direction of the spherical surface approximating the femoral head.

3-D MR images of sagittal sections were obtained using 3-D-spoiled gradientecho sequences (SPGR) [7] under the following two conditions.

- MR imaging with cubic voxels: $\Delta_{x y}=\Delta_{z}=0.7(\mathrm{~mm})$.

- Routinely performed MR imaging with noncubic voxels (routine imaging): $\Delta_{x y}=0.625(\mathrm{~mm}), \Delta_{z}=1.5(\mathrm{~mm})$.

To obtain an acceptable signal-to-noise ratio, the imaging time with cubic voxels was three times as long as that employed in routine imaging with noncubic voxels. In each $3-\mathrm{D}$ MR image, the matrix size was $256 \times 256$.

\section{Procedures for Segmentation and Quantification}

Interpolation: 3-D MR images were trimmed and then interpolated using sinc interpolation [13, 14] such that (i) the signal sampling was isotropic in all three directions and (ii) the image size was doubled. The sampling interval in the interpolated data was $0.35(\mathrm{~mm})$ in the imaging with cubic voxels and 0.3125 $(\mathrm{mm})$ in the routine imaging. Note, however, that the volume ndata obtained using the routine imaging were inherently more blurred in the $z$ direction than in the $x y$-plane even though the signal sampling was isotropic.

Cartilage Segmentation: In the real MR images, cartilage region segmentation was needed before thickness quantification. To facilitate segmentation, cartilage enhancement filtering was performed according to the following equation:

$$
I_{\text {cartilage }}(\mathbf{x})=\max _{i}\left\{-\sigma_{i}^{2} D_{2}\left(\mathbf{x} ; \sigma_{i}, \mathbf{r}\right)\right\},
$$

where $\sigma_{i}=2^{(i-1) / 2}$ (voxels) (in which $\left.i=1,2,3\right)$ and $\mathbf{r}=\frac{\mathbf{x}-\mathbf{c}}{|\mathbf{x}-\mathbf{c}|}$ (in which $\mathbf{c}$ is the center position of the sphere approximating the femoral head). In the multiscale integration of Eq.(13),$\sigma_{i}^{2}$ was multiplied for the normalization of each scale $\sigma_{i}$ [15], [16].

The filtered images $I_{\text {cartilage }}(\mathbf{x})$ were thresholded, with the threshold values being determined through operator interaction. Using connectivity analysis, the approximated segmented regions of the cartilage, $S_{\text {cartilage, }}$, were extracted.

Thickness Quantification: The extracted 3-D cartilage regions were thinned to a width of one voxel by non-maximum suppression along the radial directions of the filter-enhanced cartilage images. A cartilage thickness was assigned to each point of the thinned cartilage regions. 
For each point of the thinned regions, the profile of the directional second derivative $D_{2}(\mathbf{x} ; \sigma, \mathbf{c})$ was reconstructed along the radial directional line that passed through this point and originated from the center point, $\mathbf{c}$, of the sphere approximating the femoral head. Here, $\sigma$ was the standard deviation of the Gaussian blurring combined with the second derivative computation. Similarly, binary profiles were reconstructed along the same radial directional line for the binary images of the segmented cartilage $S_{\text {cartilage }}$.

Profile reconstruction was performed at subvoxel resolution by using a trilinear interpolation for the directional second derivative and a nearest-neighbor interpolation for the segmented cartilage. Let $D_{2}(r)$ be the profile of the radial directional second derivative, and $S_{\text {cartilage }}(r)$ be the profile of the segmented binary cartilage images (here, $r$ denotes the distance from the center of the sphere approximation, c). Cartilage edges were localized in two steps: finding the initial point for the subsequent search using $S_{\text {cartilage }}(r)$, and then searching for the zero-crossing of $D_{2}(r)$. The initial point of the outer edge, $p_{\text {out }}$, was given by the maximum value of $r$ that satisfied $S_{\text {cartilage }}(r)=1$ if it existed. Otherwise, the edge localization process terminated. The initial point of the inner edge, $p_{i n_{0}}$, was given by the minimum value of $r$ that satisfied $S_{\text {cartilage }}(r)=1$.

Given the initial point of the search, if $D_{2}\left(p_{\text {out }}\right)<0$, search inbound (the direction in which $r$ decreases) toward the center along the profile for the zerocrossing position $p_{\text {out }}$. Otherwise search outbound. Similarly, if $D_{2}\left(p_{i n_{0}}\right)<0$, search outbound along the profile for the zero-crossing position $p_{i n}$. Otherwise search inbound. The thickness, $T$, was given by $\left|p_{\text {in }}-p_{\text {out }}\right|$.

\section{Results}

\subsection{Numerical Simulations}

Sheet structures with thickness $t=1.2,1.6,2.0$, and $2.4(\mathrm{~mm})$ were modeled. Each sheet model had orientations, $\theta$, from 0 to 90 degrees. The MR imaging numerical simulations were performed using the following parameters.

- Simulating MR imaging with cubic voxels: $\Delta_{x y}=\Delta_{z}=0.7(\mathrm{~mm})$.

- Simulating routine MR imaging with noncubic voxels: $\Delta_{x y}=0.625(\mathrm{~mm})$, $\Delta_{z}=1.5(\mathrm{~mm})$.

For $\sigma$, used in postprocessing for thickness determination, the following three parameter values were employed: $0, \frac{1}{2} \Delta_{x y}$, and $\Delta_{x y}$.

Figure 1 shows the variations in the estimated thicknesses against the angles, $\theta$, of the sheet structures in simulations of MR imaging with cubic voxels. While cubic voxels have the same resolution in the three orthogonal directions along the coordinate axes, they are still not isotropic for all directions. When $\sigma=0$, considerable differences in the estimated thicknesses were observed between the directions along the coordinate axes $\left(\theta=0^{\circ}, 90^{\circ}\right)$ and the diagonal directions $\left(\theta=45^{\circ}\right)$. When $\sigma=\Delta_{x y}$, the angle dependency was reduced and the true and estimated thicknesses were well-correlated. However, when $\sigma=2 \Delta_{x y}$, although 
the angle dependency was further reduced, considerable overestimation was observed as the sheet structures became thinner. Appropriate Gaussian smoothing $\left(\sigma=\Delta_{x y}\right)$ was thus effective in reducing the angle dependency as well as in improving accuracy.

Figure 2 shows the variations in the estimated thicknesses against the angles, $\theta$, of sheet structures in simulations of MR imaging with noncubic voxels. The angle dependency was significantly large while the accuracy was sufficiently good when $\theta<25^{\circ}$. Appropriate Gaussian smoothing $\left(\sigma=\Delta_{x y}\right)$ was also effective in the case of noncubic voxels.

\subsection{Validating the Numerical Simulation by In Vitro Experiments}

Figure 3 shows the variations in the estimated cartilage thicknesses against $\theta$, which is the angular difference between the $x y$-plane of the 3-D MR image and the surface normal direction of the sphere approximating the femoral head. Figure 3(a) shows an MR slice image of the resected femoral head. The articular cartilages are imaged as bright sheet structures distributed on the spherical head surface. We used $\sigma=0.5 \Delta_{x y}$ in the thickness determination. In Fig. 3(b), thicknesses estimated in imaging with cubic voxels $\left(\Delta_{x y}=\Delta_{z}=0.7(\mathrm{~mm})\right)$ were used as reference thicknesses. The accuracy of these thicknesses was confirmed to be sufficiently high for every angle of the sheet structures from the simulation results shown in Fig. 1. The experimental results in Fig. 3 correlate well with the theoretical ones in Fig. 2.

\section{Conclusions}

The fundamental limits to the accuracy of thickness determination in MR images were investigated. A simulation method was established to derive the characteristics of thickness determination accuracy, given both imaging and postprocessing parameters. The effect of sheet structure orientation on accuracy in the case of noncubic (anisotropic) voxels was clarified through the simulation. It was also found that postprocessing (Gaussian) blurring plays an important role in realizing accurate and well-behaved thickness determination. The simulations were validated by comparison with results obtained in in vitro experiments using the cartilages of a resected femoral head.

Future work will include the formulation of a method for automatically estimating the orientation of a sheet structure and determining its thickness with a theoretically derived confidence measure. When thickness quantification is applied to in vivo MR images, the segmentation of sheet structures of interest becomes important as well as the quantification itself. We are planning to develop a unified framework for both segmentation and quantification based on multi-scale and multi-orientation 3D image analysis.

Acknowledgment This work was partly supported by JSPS Research for the Future Program JSPS-RFTF99I00903 and JSPS Grant-in-Aid for Scientific Research (C)(2) 11680389. 

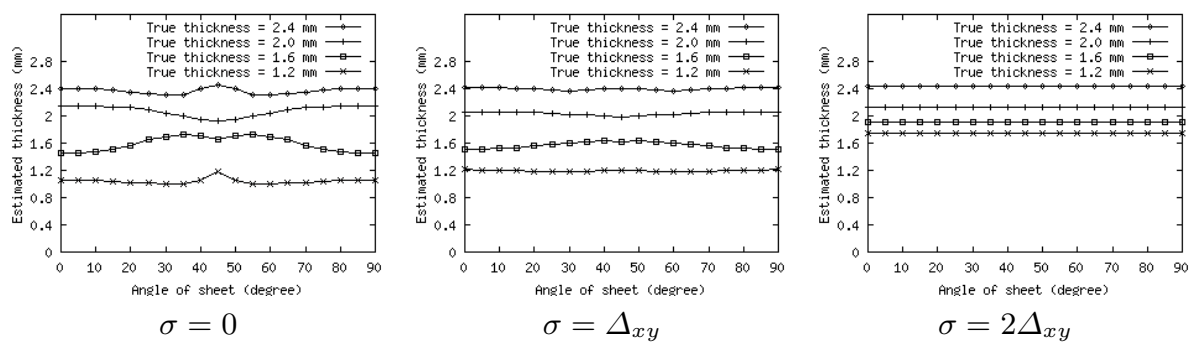

Fig. 1. Numerical simulations for thickness determination from images with cubic voxels. Variations in the estimated thicknesses against the angles $(\theta)$ of the sheet structures are shown. $\Delta_{x y}=\Delta_{z}=0.7(\mathrm{~mm})$.
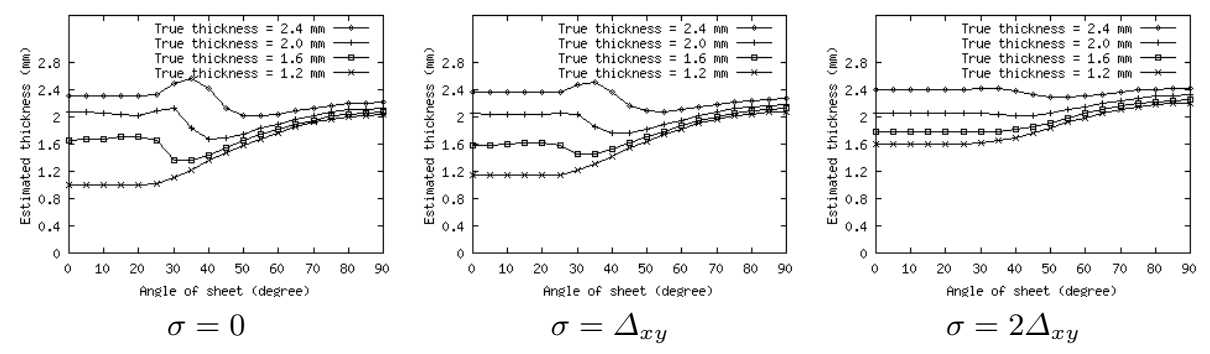

Fig. 2. Numerical simulations for thickness determination from routine MR images with noncubic voxels. $\Delta_{x y}=0.625(\mathrm{~mm}), \Delta_{z}=1.5(\mathrm{~mm})$.

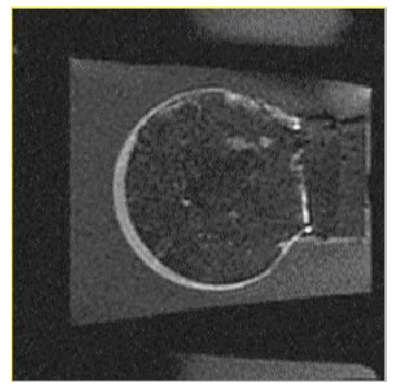

(a)

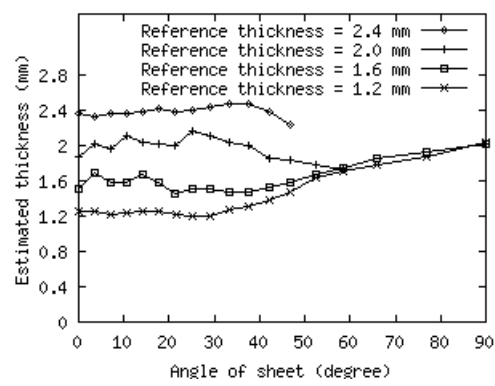

(b)

Fig. 3. In vitro experiments for thickness determination. (a) MR slice image of resected femoral head. (b) Variations in the estimated thicknesses with routine MR imaging against the angles of sheet structures. The thicknesses estimated in imaging with cubic voxels were used as reference thicknesses. 


\section{References}

1. Jonsson K, Buckwalter K, Helvie M, Niklason L, and Martel W: Precision of hyaline cartilage thickness measurements, Acta Radiol, 33, 234-239 (1992).

2. Hodler J, Trundell D, Pathria MN, and Resnick D: Width of the articular cartilage of the hip: quantification by using fat-suppression spin-echo MR imaging in cadavers, AJR Am J Roentgenol, 159, 351-355 (1992).

3. Eckstein F, Gavazzini A, Sittek H, Haubner M, Losch A, Milz S, Englmeier K-H, Schulte E, Putz R, and Reiser M: Determination of knee joint cartilage thickness using three-dimensional magnetic resonance chondro-crassometry (3D MR-CCM), Magn Reson Med, 36, 256-265 (1996).

4. Solloway S, Hutchinson CE, Waterton JG, Taylor CJ, The use of active shape models for making thickness measurements of articular cartilage from MR images, Magn Reson Med, 37, 943-952 (1997).

5. McGibbon CA, Dupuy DE, Palmer WE, and Krebs D.: Cartilage and subchondral bone thickness distribution with MR imaging, Acad Radiol, 5, $20-25$ (1998).

6. McGibbon CA, Palmer WE, and Krebs DE: A general computing method for spatial cartilage thickness from co-planar MRI, Med Eng Phys, 20, 169-176 (1998).

7. Nakanishi N, Tanaka H, Nishii T, Masuhara K, Narumi Y, and Nakamura H: MR evaluation of the articular cartilage of the femoral head during traction, Acta Radiol, 40, 60-63 (1999).

8. Sato Y, Kubota T, Nakanishi K, Sugano N, Nishii T, Ohzono K, Nakamura H, Ochi O, and Tamura S: Three-dimensional reconstruction and quantification of hip joint cartilages from magnetic resonance images, Lecture Notes in Computer Science (LNCS), 1679 (MICCAI'99), 338-347 (1999).

9. Zeng X, Staib LH, Schults RT, and Duncan JS: Segmentation and measurement of the cortex from 3-D MR images using coupled-surfaces propagation, IEEE Trans Med Imaging, 18, 927-937 (1999).

10. Magnotta VA, Andreasen NC, Schultz SK, Harris G, Cizadlo T, Heckel D, Nopoulos $\mathrm{P}$, Flaum M: Quantitative in vivo measurement of gyrification in the human brain: changes associated with aging, Cereb Cortex, 9, 151-160, 1999.

11. Parker DL, Du YP, and Davis WL: The voxel sensitivity function in Fourier transform imaging: applications to magnetic resonance angiography, Magn Reson Med, 33, 156-162 (1995).

12. Hoogeveen, RM, Bakker CJG, and Viergever MA: Limits to the accuracy of vessel diameter measurement in MR angiography, J Magn Reson Imaging, 8, 1228-1235 (1998).

13. Hylton NM, Simovsky I, Li AJ, Hale JD: Impact of section doubling on MR angiography, Radiology, 185, 899-902 (1992).

14. Du YP, Parker DL, Davis WL, Cao G: Reduction of partial-volume artifacts with zero-filled interpolation in three-dimensional MR angiography, J Magn Reson Imaging, 4, 733-741 (1995).

15. Sato Y, Nakajima S, Shiraga N, Atsumi H, Yoshida S, Koller T, Guido G, and Kikinis R: Three dimensional multi-scale line filter for segmentation and visualization of curvilinear structures in medical images, Med Image Anal, 2, 143-168 (1998).

16. Sato Y, Westin C-F, Bhalerao A, Nakajima S, Shiraga N, Tamura S, and Kikinis R: Tissue classification based on 3D local intensity structures for volume rendering, IEEE Transactions on Visualization and Computer Graphics, 6, 160-180 (2000). 